\title{
Corrigendum
}

\section{Evaluating intervention strategies in controlling coronavirus disease 2019 (COVID-19) spread in care homes: An agent-based model - CORRIGENDUM}

Le Khanh Ngan Nguyen, Susan Howick, Dennis McLafferty, Gillian H. Anderson, Sahaya J. Pravinkumar, Robert Van Der Meer and Itamar Megiddo

In the above article ${ }^{1}$, the author names should appear as follows:

Le Khanh Ngan Nguyen, Susan Howick, Dennis McLafferty, Gillian H. Anderson, Sahaya J. Pravinkumar, Robert Van Der Meer and Itamar Megiddo

\section{Reference}

1. Nguyen, L., Howick, S., McLafferty, D., Anderson, G., Pravinkumar, S., Van Der Meer, R., \& Megiddo, I. (2020). Evaluating intervention strategies in controlling coronavirus disease 2019 (COVID-19) spread in care homes: An agent-based model. Infection Control \& Hospital Epidemiology, 1-11. doi: 10.1017/ice.2020.1369 